\title{
Application of digital image correlation to address complex motions in thermoelastic stress analysis
}

\section{W Wang, R K Fruehmann, J M Dulieu-Barton*}

Faculty of Engineering and the Environment, University of Southampton, Highfield, Southampton, SO17 1BJ, UK

*Corresponding author Email: janice@soton.ac.uk

\begin{abstract}
:
A motion compensation method for thermoelastic stress analysis (TSA) is described that uses digital image correlation (DIC) to capture the displacement field on the surface of the specimen. The displacement field is used to correct the infrared (IR) images to remove the effect of the motion of the specimen from the TSA. As the DIC displacements are obtained with a relatively high spatial resolution, sharp displacement gradients and discontinuities can be corrected. The feasibility of the motion compensation method for TSA is investigated firstly by validating the approach using data obtained from an aluminium alloy plate with a central circular hole loaded in tension and comparing the results with a finite element model. It is shown that the motion compensation approach significantly improves the accuracy of TSA, particularly when high magnification optics are used. Next, the feasibility of simultaneous capture of IR and white light images is investigated. It is shown that by using the correct combination of paints, a speckle pattern can be applied to the surface to provide contrast in the white light spectrum for the DIC, but have a uniform emissivity in the IR spectrum so that there is no effect on the TSA. Thus it is possible for the motion compensation to be conducted on data collected during fatigue tests. Finally, it is demonstrated that the motion compensation technique can be applied to discontinuous motion produced by face sheet debonding in a foam cored sandwich structure loaded in a double cantilever beam (DCB) configuration. It is shown that the motion compensation technique is capable of correcting the complex and non-uniform motion for TSA in the DCB test, thereby enabling detailed thermoelastic data to be obtained from the vicinity of the crack tip.
\end{abstract}

Keywords: Thermoelastic stress analysis (TSA), Motion compensation, Digital image correlation (DIC). 
Wang W., Fruehmann, R.K. and Dulieu-Barton, J.M., "Application of digital image correlation to address complex motions in thermoelastic stress analysis", Strain, 51, 2015, 405-418. doi: 10.1111/str.12151

\section{Introduction}

Thermoelastic stress analysis (TSA) [1] is a non-contacting and full-field technique which has been widely used for evaluating stresses on the surface of components and structures. The technique is based on the thermoelastic effect where a reversible temperature change induced by cyclic loading can be directly related to the change in stresses [2]. In TSA it is usual to obtain the surface temperature change using an infrared (IR) detector. Since very high resolution data can be obtained directly from the vicinity of a defect, this technique has proven to be a useful tool to evaluate the local effects (e.g. damage).

In many scenarios, large and discontinuous rigid body motion occurs during testing. Motion of the specimen, relative to the stationary IR detector, leads to blurring of the data and erroneous assessment of the temperature changes locally on the specimen surface. For this reason, it is essential to correct for the motion. It has been suggested that for uncooled detectors ${ }^{*}$, the effect of motion can be reduced by attaching the detector to the structure so that there is no relative motion between the two. However, the cryogenic cooling system and the electronics associated with analogue to digital conversion are housed inside most modern cameras making the system heavy which could result possible undesirable inertial effects and damage to the camera.

Motion compensation methods can be catagorised into those that use some form of marker tracking, and those that use digital image correlation (DIC) [3] to obtain displacement fields. A further distinction can be made between methods that use images from the IR camera for determining the motion fields (one camera methods) and those that use a separate camera system (e.g. white light camera) for determining the motion fields (two camera methods). Sakagami et al. [4] and Silva and Ravichandaran [5] are examples where a one camera DIC method was employed to correct for specimen motion in thermal images for TSA. In both publications, a single IR detector was used to obtain the data for both the TSA analysis and the DIC based motion correction. The use of an IR detector for DIC requires the specimen to be painted using paints with different emissivity values in the IR spectrum to form a speckle pattern. However, the variation of surface emissivity makes the assessment of

\footnotetext{
* Private communication with Professor Robert Rowlands, University of Wisconsin, USA
} 
Wang W., Fruehmann, R.K. and Dulieu-Barton, J.M., “Application of digital image correlation to address complex motions in thermoelastic stress analysis", Strain, 51, 2015, 405-418. doi: 10.1111/str.12151

the surface temperature difficult. To circumvent the effect of the speckle pattern to TSA, in reference [4] the specimen motion was obtained first using a separate set of IR images for DIC. Then the speckle was removed and a second data set was collected for TSA. Removal of the painted speckle pattern in-situ in the load machine presents a challenge, as does the precise replacement of a specimen that has been removed for repainting. Also, some rough surfaces make complete removal and uniform repainting nearly impossible. In [5] both the DIC and TSA were performed on the same IR images. The gaps in the data arising from the speckles were filled by interpolating across the speckles. This requires the speckles to be relatively sparsely distributed, and lowers the spatial resolution of the displacement field obtained by DIC. The use of a single IR detector to capture both images for DIC and TSA has the benefit that the images collected possess the same pixel resolutions and no spatial alignment is required. However, images obtained from current IR detectors can provide only limited spatial resolutions, typically no more than $640 \times 512$ pixels in one image, and significantly reduce the resolution of the displacement field obtained by the DIC. This does not pose a problem when the motion is relatively uniform across the surface of the specimen. However, when the motion of the structure changes rapidly and discontinuously across the field of view, as in the case of a face sheet debonding from a sandwich structure, addressed in the present paper, the low detector spatial resolution prevents the details of the motion to be captured, thereby resulting in a decrease in accuracy of the DIC. Instead of using DIC, Pottier et al. [6] applied a number of reflective points (spots) on the specimen surface before the test to track the specimen motion. The motion of each pixel in the IR image was determined by obtaining the displacements of the three spots closest to the pixel using a linear shape function. The method is time consuming as that the location of each spot has to be determined manually. This makes it both user dependent, and unsuited to cases where there are sharp displacement gradients requiring a greater density of spots. Furthermore, as with the previous methods, the spots mask the thermal data and reduce the resolution available for TSA. Thus, this method is unsuitable for situations where the specimen motion changes rapidly and discontinuously.

The double cantilever beam (DCB) test [7] is used to characterise the interfacial debonding of sandwich beams (or laminates) under mode I loading conditions. TSA 
can be used to assess the stress state around the crack tip of the DCB test specimen, opening the possibility of deriving the crack-tip parameters during interfacial debonding as demonstrated for homogeneous materials in [8] and [9]. However, the motions near the crack tip in a DCB specimen are complex, so it is necessary to develop an appropriate motion compensation approach for cases where the motion is complex before an analysis of the crack-tip can be performed.

There have been several studies that use conventional white light cameras to perform DIC to correct for motion in IR images. In [10-12] a beam splitter was used to separate the white light and IR radiation, to enable both cameras to capture images from the specimen surface simultaneously. However, because of distortion through the beam splitter the two cameras cannot observe exactly the same zone on the specimen surface. Therefore it is necessary to align the IR images with the white light images. This was done using three indentations on the specimen surface. A relationship was established that describes the transformation between the two coordinate systems, which allows the location of each pixel in the IR to be identified in the white light image, hence establishing the displacements of the IR image. However, the method has not been applied in a situation where cyclic loading is used. In [13-15] the images for DIC and the IR were obtained from the sides of the specimen, at the same time, using a trigger system. The IR images captured as the specimen deformed were mapped to a reference coordinate system using the displacement data obtained from the DIC. Although this method can be applied to cyclic loading, it assumes that the displacement on both sides of the specimen are exactly the same, which may not be valid in many situations particularly when the specimen is thick.

The present work aims to develop a motion compensation method for TSA that addresses firstly, the spatial resolution issues associated with IR reflective markers, so that it can be applied to complex motion fields and secondly, is suited to cyclic loading and fatigue testing. The proposed method is based on DIC using a high resolution white light sensor. The displacement field is then used to correct for the effect of motion in the IR images. To begin with, a description of the test arrangements and the data processing approach for the motion compensation method is provided. The method is then tested using a uniform matt black paint coating for the 
TSA, and subsequently applying a speckle pattern on top of the black coating for the DIC. For validation, the results with and without motion compensation are compared with finite element (FE) model results. The second test aims to assess the feasibility of capturing the images for TSA and DIC simultaneously, i.e. using the same surface preparation for both the TSA and the DIC. TSA is performed on the same specimen, but without removing the speckle pattern, and the results are validated against the first test. Finally, the motion compensation technique is applied to IR data obtained from a DCB test to demonstrate that it can successfully account for complex specimen motion.

\section{Methodology}

The challenge of addressing test specimen motion arises whenever a change in stress is required to effect a measurement made using an imaging system, as usually the imaging detector is stationary and the specimen moves relative to the sensor. In TSA the measuring device is typically a cryogenically cooled IR detector, which is used to observe a change in temperature on the surface of a cyclically loaded component. The component will therefore move relative to the image, as shown schematically in Fig.1 for the example of an arbitrary sample undergoing sinusoidal loading. The change in temperature of the top right corner of the specimen (Pixel 1 in Fig.1) between the maximum and minimum values in the load cycle is $T_{2}-T_{1}$. However, since the analysis is conducted on a pixel by pixel basis, the detector will output $T_{3}-T_{1}$. Specimen motion is considered significant when the area of interest includes large stress gradients and when motion exceeds 1 image pixel, although the effect can be observed for displacements smaller than 1 image pixel at specimen edges.

In DIC, the images collected before and after deformation are divided into small areas called subsets. The motion of each subset is tracked using a correlation criterion, and the displacement vector of each subset is then computed, thereby providing a map of the displacement field [3]. By incorporating the displacement fields into the IR images, the motion of each pixel in the IR image can be corrected. This forms the fundamental principle of the DIC based motion compensation technique. 
Wang W., Fruehmann, R.K. and Dulieu-Barton, J.M., "Application of digital image correlation to address complex motions in thermoelastic stress analysis", Strain, 51, 2015, 405-418. doi: 10.1111/str.12151

\subsection{Test arrangements}

The motion compensation technique captures the images for TSA and DIC as follows:

1. The specimen is coated with matt black paint to increase and homogenise the surface emissivity.

2. As images are acquired by two separate camera systems, for alignment purposes it is necessary to apply position marks on the specimen, which have a strong contrast in both the IR and white light spectra.

3. The specimen is mounted into a test machine capable of cyclic loading.

4. The temperature time history is obtained by the IR detector during cyclic loading so that it can be used in the TSA.

5. Without removing the specimen from the machine, a random white speckle pattern is applied on top of the matt black coating for DIC.

6. White light images are captured at a rate of $1 \mathrm{~Hz}$ as the specimen is loaded quasi statically at rate of $1 \mathrm{~mm} / \mathrm{min}$ over a range equivalent to the dynamic loading range used in step 4.

The IR detector system used in this work is a Cedip Silver 480M system with a sensitivity of $4.2 \mathrm{mK}$ at $25^{\circ} \mathrm{C}$ and a resolution of $320 \times 256$ pixels. The IR data were recorded with a frame rate up to $383 \mathrm{~Hz}$. For the white light imaging a LA Vision VCImager E-lite digital camera with a 5 mega-pixel sensor array was used. The use of the two camera systems requires the white light and IR images collected from the specimen surface to be aligned as the cameras may rotate (both in-plane and out-ofplane) relative to the specimen surface. To facilitate alignment of the IR images and the DIC displacement map, a specimen edge was used to provide a datum for in-plane rotation alignment. When a magnifying lens is used, the edge of the position marks is used for the same purpose. To minimise out-of-plane misalignment (i.e. to ensure that the imaging plane is parallel to the specimen surface) a spirit level was placed perpendicular to the specimen surface pointing towards the camera, and the camera position was adjusted to be perpendicular with the spirit level. Parallax errors are accounted for by alignment of the cameras perpendicular to the surface of the specimen and by choosing the same image centre (in the present work, the cameras 
are not operated side by side where parallax would be an important consideration). Lens aberrations are most significant near the edges of an image and are also dependent on the size of the detector relative to the focal length of the lens system. In the present work, the area of interest focused on an area away from the image edges. Ideally both cameras would be presented with a $3 \mathrm{D}$ calibration object to enable lens aberrations to be fully corrected. This was not done in the current work and is an area for improvement.

\subsection{Data processing}

The displacement of the specimen was derived using the DaVis 7.4 software produced by LA Vision. It is necessary to interpolate the displacement vector fields to fit to the IR data as the sensor arrays of the cameras are different and hence the spatial resolution of the IR and the displacement data are different. To make the data sets comparable, a linear interpolation procedure was applied to the displacement field. Fig. 2 shows the interpolation process for deriving the displacement vector $(D)$ of one pixel in the IR data. The global image datum origin is at the top left corner of the white light image. The lower right corner of each pixel in the IR image is used as the reference datum for the interpolation. As the IR and white light images have been aligned by the position marks, the location of the IR datum in the displacement field can be determined according to the distance between the IR datum and position marks. The interpolation of the displacement field was performed using a bilinear interpolation, i.e. by firstly conducting the interpolation in the x-direction and then the y-direction. Referring to Fig. 2 the horizontal component $(U)$ of the displacement vector $(D)$, from now on referred to as the horizontal displacement, is interpolated in the $\mathrm{x}$-direction along the top and bottom of the cell relative to the IR datum as follows:

$$
\begin{aligned}
& U_{i+\Delta l_{x}, j}=\frac{d-\Delta l_{x}}{d} U_{i, j}+\frac{\Delta l_{x}}{d} U_{i+1, j} \\
& U_{i+\Delta l_{x}, j+1}=\frac{d-\Delta l_{x}}{d} U_{i, j+1}+\frac{\Delta l_{x}}{d} U_{i+1, j+1}
\end{aligned}
$$

where $U_{i, j}$ represents the horizontal displacement at the mid-point of the DIC subset $(i, j), \Delta l_{x}$ is the horizontal (x-direction) distance between an adjacent point on the 
displacement grid and the IR datum, and $d$ is the distance between the centres of adjacent DIC subsets. The horizontal displacements given by equations (1) and (2) are then interpolated in the vertical (y-direction) to provide the horizontal displacement of each pixel in the IR image as follows:

$U_{i+\Delta l_{x}, j+\Delta l_{y}}=\frac{d-\Delta l_{y}}{d} U_{i+\Delta l_{x}, j}+\frac{\Delta l_{y}}{d} U_{i+\Delta l_{x}, j+1}$

where $\Delta l_{y}$ is the vertical distance between an adjacent point on the displacement grid and the IR datum.

The same process is used to obtain the vertical component $(V)$ of the displacement vector. $U$ and $V$ are then combined to give $D$, shown by the arrows in Fig. 2, as follows:

$$
D_{i+\Delta l_{x}, j+\Delta l_{y}}=U_{i+\Delta l_{x}, j+\Delta l_{y}}+V_{i+\Delta l_{x}, j+\Delta l_{y}}
$$

The images for the DIC were captured during quasi-static loading over a range equivalent to that of the cyclic load in the IR data capture. This gives the displacement vectors at the extremes of the load cycle. However, the IR data are sampled at many points throughout the loading cycle. Hence, the image displacement correction needs to be interpolated between the maxima and minima depending on the time of IR image capture within the loading cycle. For this it is assumed that the loaddisplacement relationship is linear. The displacement at each frame can then be calculated as a proportion of the maximum displacement for each pixel in the IR image. The position of an IR image in the loading cycle is obtained from the load cell signal recorded synchronously with each IR image, i.e. the IR detector system incorporates a reference signal input that is recorded with each IR image. This reference signal is scaled to vary between zero and one, shown schematically in Fig. 3 (blue line); i.e. the IR image at the minimum load is defined as the zero displacement image over which all other IR images are overlaid. A corresponding displacement curve can then be generated for each IR image pixel represented by the black line in Fig. 3. These displacement curves are calculated for each IR image pixel according to:

$$
D=D_{\max }\left[\left(\sin \left(\theta n+\frac{3}{2} \pi\right)+1\right) / 2\right]
$$


where $D_{\max }$ is the displacement vector interpolated at each pixel in the IR image when the maximum load is applied, $\theta$ is the interval between IR images expressed as an angle in radians such that $\theta=2 \pi f_{l} / f_{r}$, where $f_{l}$ is the loading frequency and $f_{r}$ is the frame rate of the IR detector (both in $\mathrm{Hz}$ ) and $n$ is the image number.

According to the computed displacement field, the position of each pixel can be redefined for each frame, and a series of corrected IR images can be constructed. The corrected pixel position is rounded to the nearest integer (i.e. sub-pixel displacements and associated temperature interpolations have been omitted as their effect was found to be negligible in the current work). The magnitude of the temperature change for each pixel is obtained from the motion compensated temperature time history using a fast Fourier transform (FFT) method. For the purpose of computational efficiency, the corrected IR and interpolated displacement images are not stored. Instead, each IR image pixel is processed in turn, a corrected temperature-time series is constructed and input into a FFT. The magnitude and phase values at the loading frequency are then stored in two output images; one for the magnitude data and one for the phase data. The phase is useful as it enables the identification of compressive and tensile stresses, with a $180^{\circ}$ shift between the two. Moreover, it is also used to determine if the response is being influenced by factors other than the thermoelastic coupling (e.g. heat transfer by conduction and dissipation). The method incorporates the collection of many white light images during the deformation hence large displacements can be handled as long as they are in the linear elastic region, i.e. that which is valid for TSA.

\section{Experimental validation}

\subsection{Experimental arrangements}

The feasibility of the motion compensation technique is demonstrated by correcting for the motion from TSA data captured around the edge of a circular hole in an aluminium alloy specimen. The material properties of the aluminium alloy were obtained experimentally and are shown in Table 1. The configuration of the specimen is shown in Fig. 4 (a). The specimen was $2 \mathrm{~mm}$ thick by $50 \mathrm{~mm}$ wide with a length of $600 \mathrm{~mm}$. An $8 \mathrm{~mm}$ diameter hole was produced using electro-discharge machining 
and located $190 \mathrm{~mm}$ from the end of the specimen, i.e. closest to the actuator of the servo hydraulic test machine. This was done to ensure sufficient motion, i.e. greater than an IR image pixel, around the hole during cyclic loading. The tests were conducted using an Instron 8802 servo hydraulic test machine (fitted with a $100 \mathrm{kN}$ actuator and load cell). A cyclic load with $4.2 \mathrm{kN}$ mean load and $3.4 \mathrm{kN}$ load amplitude, with a frequency of $15 \mathrm{~Hz}$, was applied to the specimen, also to generate motion around the hole which is large enough to be observed in the IR images. However, a simple calculation based on an stress concentration factor of 3 shows that this load level will generate some plasticity in the neighbourhood of the hole (Table 1 provides the yield stress of the material). It was considered more desirable to generate a loading that would provide a measurable displacement rather than to reduce the load and avoid the plasticity.

Table 1. Material properties for aluminium specimen

\begin{tabular}{lccc}
\hline \hline & $\begin{array}{c}\text { Elastic modulus } \\
(\mathrm{GPa})\end{array}$ & $\begin{array}{c}\text { Poisson's } \\
\text { ratio }\end{array}$ & $\begin{array}{c}\text { Yield strength } \\
(\mathrm{MPa})\end{array}$ \\
\hline Aluminium alloy & 70 & 0.33 & 110 \\
\hline \hline
\end{tabular}

Prior to testing, the specimen was coated with two passes of RS matt black paint. The paint is required to provide a suitably high emissivity. As suggested in [16], the paint was applied so as to be in the range of 15 to $25 \mu \mathrm{m}$, which was confirmed using a Thikstik Dual Gauge produced by Sheen Instruments. Several position marks comprising reflective metal tape were placed on the coated specimen surface as shown in Fig. 4 (b) to align the IR and DIC images. In both IR and white light images, the areas bounded by the position marks were processed.

The IR images were taken using a $27 \mathrm{~mm}$ lens to conduct the motion compensation at a relatively large scale with a resolution of $0.24 \mathrm{~mm} /$ pixel. A magnifying lens was also used, referred to by the manufacturer as a G1 lens, to evaluate the effectiveness of the motion compensation at greater magnifications with a resolution of 0.03 $\mathrm{mm} /$ pixel. The measurement areas on the specimen of each of these two lenses are shown in Fig. 4 (b). 
Wang W., Fruehmann, R.K. and Dulieu-Barton, J.M., “Application of digital image correlation to address complex motions in thermoelastic stress analysis", Strain, 51, 2015, 405-418. doi: 10.1111/str.12151

The speckle pattern required for DIC was generated by spraying Ambersil matt white paint randomly onto the black coating. To achieve a similar field of view to that of the IR data capture, two different lenses were used to capture the white light images. A $105 \mathrm{~mm}$ lens (SIGMA) was set with a scale factor of $0.018 \mathrm{~mm} /$ pixel to provide a measurement area $\left(45 \times 37 \mathrm{~mm}^{2}\right)$ comparable to $27 \mathrm{~mm}$ lens. A $65 \mathrm{~mm}$ macro lens (CANON MP-E) provided a field of view comparable to the G1 lens $\left(8 \times 7 \mathrm{~mm}^{2}\right.$ with a scale factor of $0.0033 \mathrm{~mm} / \mathrm{pixel}$ ). The specimen displacement was measured over the same loading range (from $0.8 \mathrm{kN}$ to $7.6 \mathrm{kN}$ ) as used for the collection of the IR images.

\subsection{Analysis of specimen displacement}

In DIC the accuracy of image correlation is highly dependent on the subset size [17]. A larger subset size increases the uniqueness for the correlation as more speckles are considered, thereby offering better displacement precision, but increasing computing time and reducing spatial resolution. The DaVis 7.4 software used in this work provides a wide range of subsets in powers of two from $16 \times 16$ to $512 \times 512$ with an overlap from $0 \%$ to $99 \%$. To select an appropriate subset size, the displacement field extracted from the recorded images of two different lenses were computed using three different subset sizes: $32 \times 32$ pixels, $64 \times 64$ pixels and $128 \times 128$ pixels. The displacements at maximum load $(7.6 \mathrm{kN})$ along the white dashed line in the inserts in Fig. 5 were calculated for each of the three subset sizes and compared. The origin of the coordinate system is set at the centre of the hole; this coordinate system was used in all the plots in sections 3 and 4 . It was found that the results from $64 \times 64$ and 128 $\times 128$ exhibit almost the same trend, while the results from $32 \times 32$ present significant fluctuation. As the large subset size provides less spatial resolution, $64 \times 64$ is selected here as the optimum subset size. In Fig. 5, the displacement derived from DIC shows an approximate $0.4 \mathrm{~mm}$ displacement at the edge of the hole. When comparing to the pixel size in the IR image, this displacement will introduce a 2 pixel movement when using the $27 \mathrm{~mm}$ lens and more than 10 pixels movement when the G1 lens is used. 
Wang W., Fruehmann, R.K. and Dulieu-Barton, J.M., "Application of digital image correlation to address complex motions in thermoelastic stress analysis", Strain, 51, 2015, 405-418. doi: 10.1111/str.12151

\subsection{Results}

To enable a quantitative evaluation of the stress values obtained from the TSA, the thermoelastic constant is required for the aluminium material. A value of $9.61 \times 10^{-12}$ $\pm 0.16 \mathrm{~Pa}^{-1}[9]$ was used to calibrate the TSA data. To account for localised plasticity around the hole a 2D elastic-plastic FE model of the specimen was constructed using ANSYS FE software version 12.1 [18]. A stress strain curve for the material was obtained experimentally to provide input into the model to describe the material plastic behaviour. A five point multilinear kinematic hardening plasticity model was used. The FE mesh and the boundary conditions are shown in Fig. 6. The mesh is very refined in the neighbourhood of the hole, hence 2-D 4-node structural solid elements (PLANE182) were used with the material properties as given in Table 1.

The TSA results obtained with the $27 \mathrm{~mm}$ lens before and after motion compensation are shown in Fig. 7 (MC refers to motion compensation in all figures). In Fig. 7 (a) the edge effect caused by calculations of the temperature difference between the specimen surface and the background is observed. The phase data in Fig. 7 (c) also shows the edge effect. Fig. 7 (b) shows that the motion compensation enables data to be obtained close to the edge of the hole. A second artefact of motion, most noticeable in the lower half of the image in Fig. 7 (a), is due to the surface roughness of the specimen. The emissivity quoted for RS matt black paint 0.92 [16]. It is assumed to be spatially uniform across the specimen. Since transmitted radiation is assumed negligible, this gives a reflectivity of 0.08 ; i.e. $8 \%$ of any incident radiation is reflected. The surface texture causes this reflected radiation to have different sources. Where the surface is perpendicular to the line of sight of the detector, $8 \%$ of the detectors own emissions (76K) are reflected back. In other locations on the specimen surface $8 \%$ of the background emissions $(290 \mathrm{~K})$ are reflected into the detector because the surface is not perpendicular to the line of sight. At low spatial resolutions, this texture is mostly averaged within a single pixel, but a slight effect is still visible in the processed data. Once motion compensation has been applied, the reflected portion of the temperature measurement is a constant offset at each measurement point (i.e. each point on the specimen surface is always reflecting the same background source) and hence has a negligible influence on the FFT processing which evaluates only with the sinusoidal component of the signal. Fig. 7 (d) shows the 
motion compensated phase data, although there is a visible improvement in the data quality an artefact of the motion is still present, i.e. the light coloured band around the edge of the hole.

To provide a quantitative comparison of the data sets before and after motion compensation, line plots along the dashed line in Fig. 7 are shown in Fig. 8. Little difference can be seen in the data before and after motion compensation, especially away from the edge of the hole. This is because the motion has little effect when the stress field is relatively uniform. The scatter is, however, slightly larger without motion compensation due to the surface texture effect. The results after motion compensation show good agreement with the FE results. The discrepancy between the TSA and FE results very close to the edge of the hole results from the localised plasticity modifying the thermal response and hence the calibrated stress values. A further, and possibly more important, influence is the large stress gradient in the neighbourhood of the hole, so heat transfer within the specimen cannot be entirely eliminated. This is confirmed by the phase data (see Fig. 8 (b)) where the heat diffusion leads to a phase shift coincident with change in the stress gradient. The influence of the plasticity and the heat transfer on the response warrants further study but is outside the scope of the present paper where the focus is on developing the motion compensation technique. For this purpose the motion compensation approach is demonstrated by the significant improvement in the experimental data.

The effect of motion is more significant when the G1 lens is used. The TSA data adjacent to the hole before motion compensation are shown in Fig. 9 (a). The specimen motion can be observed by a large shift of the position marks, the two regions of very large stress where the hole edge curves towards the horizontal and the effect of the surface texture (barely visible in the raw IR images), that completely masks the stress field, causing the entire image to appear blurred. After employing the motion compensation method (Fig. 9 (b)), a clear stress gradient in the vicinity of the hole can be observed. The profile along the edge of the hole can be clearly identified which indicates that the influence of the motion has been significantly reduced. The data along the line marked in Fig. 9 is plotted alongside the FE results and with the TSA data obtained with the $27 \mathrm{~mm}$ lens in Fig. 10. There is a very large scatter in the data collected with the G1 lens before motion compensation, which is significantly 
Wang W., Fruehmann, R.K. and Dulieu-Barton, J.M., “Application of digital image correlation to address complex motions in thermoelastic stress analysis", Strain, 51, 2015, 405-418. doi: 10.1111/str.12151

reduced after the motion compensation was applied. The results obtained from the G1 lens after motion compensation are similar to the results obtained from the $27 \mathrm{~mm}$ lens.

\section{Influence of the speckle pattern on TSA}

The work in the previous section validated the proposed motion compensation approach using a best practice surface preparation for the DIC and the TSA. This presupposes an unchanging field between conducting the TSA and the DIC. However in many situations, such as fatigue testing it would be desirable to collect the IR images for the TSA and the images for the DIC at the same time. This would require a single coating that can be used for both the TSA and the DIC. The purpose of the present section of the paper is to show that a speckle pattern can be applied that provides sufficient contrast for the DIC but does not influence the TSA. This is possible as the emissivity in the white light and IR spectra are not necessarily the same. Hence, if a suitable combination of black and white paint is used, IR images can be collected without any influence of the speckle pattern. Tests were conducted using the same aluminium specimen as used in the first set of tests.

The test specimen was painted with both the black coating and the black and white speckle pattern. Data were collected from the vicinity of the hole using the G1 lens to magnify any influence of surface irregularities. The speckle pattern used in this work is shown in Fig. 11. The size and number of the speckles in the speckle pattern were assessed using the edge detection method described in [19]. Fig. 12 shows the frequency distribution of the speckle sizes present within the pattern. The predominant speckle size is $0.2 \mathrm{~mm}^{2}$ which is equivalent to 60 pixels per speckle at high resolution. The pattern has a speckle density of about 36 speckles per $\mathrm{mm}^{2}$.

The IR images captured from the specimen without and with the speckle pattern are shown in Fig. 13 (a) and (b) respectively. It can be seen that the IR images show a similar temperature distribution, with almost no discernible differences that can be attributed to the speckle pattern shown in Fig. 11. This indicates that the speckle pattern used in this work has almost the same emissivity as that of the matt black coating, although this has not been explicitly evaluated. The proposed motion 
Wang W., Fruehmann, R.K. and Dulieu-Barton, J.M., “Application of digital image correlation to address complex motions in thermoelastic stress analysis", Strain, 51, 2015, 405-418. doi: 10.1111/str.12151

compensation method was applied to the IR images; Fig. 13 (c) and (d) show the stress sum amplitude around the edge of the hole obtained without and with the speckle pattern respectively. In both images the stress distributions around the edge of the hole present a comparable geometry and magnitude. To provide a quantitative comparison of the two data sets, the data along the line marked in Fig. 13 (c) and (d) are plotted and compared with the FE results in Fig. 14. Little difference can be observed from the stress data collected from the black coating and the speckle pattern. This shows that the speckle pattern used in this work has almost no influence on the TSA data. Thus, the possibility to perform the measurements for TSA and DIC from the same specimen surface preparation is confirmed.

\section{Thermoelastic response of the double cantilever beam sandwich specimen}

The major motivation for developing the high spatial resolution motion compensation technique is to correct a discontinuous and complex motion field. In this case it is essential to derive high spatial resolution displacement data to accurately resolve the motion. As described in the introduction, face sheet debonding from a sandwich structure is an example where the motion in the vicinity of the debond changes rapidly and discontinuously. To demonstrate that the proposed motion compensation method can correct complex motion, the motion compensation technique is applied to a sandwich specimen loaded in a DCB configuration.

A sandwich panel was manufactured in a single shot resin infusion process using a 25 mm thick PVC foam core (DIAB H100) and E-glass/epoxy composite face sheets. The face sheet comprised eight layers of plain woven, $210 \mathrm{gm}^{-2}$ E-glass textile, giving a face sheet thickness of $1.6 \mathrm{~mm}$. A thin Teflon film $(25 \mu \mathrm{m})$ was placed between the face sheets and the core to create a controlled debond region. DCB test specimens of $200 \mathrm{~mm}$ length and $32 \mathrm{~mm}$ width were cut from the panel and loaded as in Fig. 15. The test was conducted in an Instron E1000 test machine with a $1 \mathrm{kN}$ actuator and load cell capacity at $3 \mathrm{~Hz}$ loading frequency. The test was conducted in displacement control with an amplitude of $1 \mathrm{~mm}$, giving an approximate load amplitude of $30 \mathrm{~N}$ at a frequency of $3 \mathrm{~Hz}$. The hinges (see Fig. 15) were adhesively bonded to the specimens and clamped in the test machine using standard mechanical grips. 
Wang W., Fruehmann, R.K. and Dulieu-Barton, J.M., "Application of digital image correlation to address complex motions in thermoelastic stress analysis", Strain, 51, 2015, 405-418. doi: 10.1111/str.12151

IR images were collected from the side of the specimen using both the $27 \mathrm{~mm}$ lens and G1 lens as shown in Fig. 16. The fields of view with the setup of the $27 \mathrm{~mm}$ and G1 lens were approximately $80 \times 60 \mathrm{~mm}^{2}$ and $10 \times 8 \mathrm{~mm}^{2}$. The $105 \mathrm{~mm}$ and $65 \mathrm{~mm}$ lens were used to provide a comparable measurement area for the $27 \mathrm{~mm}$ and G1 lens respectively. The area of interest of the $27 \mathrm{~mm}$ lens is indicated by the black dashed line in Fig.16 (a) which is bounded by the specimen edge and the position marks. The area is located on the specimen surface as indicated by the red line shown in Fig. 15. The G1 lens provided a field of view in the neighbourhood of the crack tip. The areas of interest associated with the G1 lens are indicated by the black dashed line in Fig. 16 (b) which is bounded by the three position marks. In Fig. 16 (a) and (b) the areas of interest are divided into two regions: an upper region which contains the upper (partly debonded) face sheet, and a lower region that comprises the foam core and the lower fully attached face sheet (just the foam core in the image taken with the G1 lens). As there is a structural discontinuity caused by the interfacial crack, the motion compensation method was applied to the upper and lower regions separately. The fullfield images were then combined using MATLAB.

The specimen displacement across the face sheet/core interface obtained by DIC is shown in Fig. 17. The vector arrows indicate the magnitude and direction of the displacement field. A large variation in displacement across the area of interest is clearly shown. The discontinuity in the displacement field necessitates the high resolution images provided by the white light camera.

The stress state around the crack tip obtained from the TSA is shown in Fig. 18 and is presented as a non-dimensional stress metric $(\Delta T / T)$, which is directly proportional to the stresses. The results are not presented as stresses since the thermoelastic constants for the orthotropic face sheet and core materials are required. As the focus of the work is development of the motion compensation routine it was considered unnecessary to embark on a laborious material characterisation exercise as described in [20]. The results were adjusted for tension and compression by multiplying the amplitude data $(\Delta T)$ by \pm 1 in accordance with the phase data. Fig. 18 (a) shows the $\Delta T / T$ data before applying the motion compensation technique. At first glance, the data appears to be reasonable with the stress gradient showing in the face sheet and a stress concentration at the crack-tip. There is some indication of the edge effect at the 
Wang W., Fruehmann, R.K. and Dulieu-Barton, J.M., "Application of digital image correlation to address complex motions in thermoelastic stress analysis", Strain, 51, 2015, 405-418. doi: 10.1111/str.12151

debonded part of the upper face sheet and a kind of shadowing of the stress concentration. The only clear indication that motion may be affecting the data is the blurred position markers in comparison to what is observed in the stationary IR image in Fig. 16 (a). The TSA results after applying the motion compensation technique are shown in Fig. 18 (b), by comparing the image in Fig. 18 (b) with that of Fig. 18 (a) the effect of motion on the TSA data becomes apparent. A clear through-thickness stress gradient from compression to tension can be observed in the upper face sheet. The cellular structure of the foam clearly leads to small variations in the reflected radiation and hence a textured image; following motion compensation a clear $\Delta T / T$ gradient is observed in the foam. The localised increase in $\Delta T / T$ in the face sheet at the end of the debond region, i.e. the crack-tip, is clear in Fig. 18 (b). Furthermore, line plots of $\Delta T / T$ along $\mathrm{x}$-direction taken $1 \mathrm{~mm}$ below the face sheet/core interface (see the black line in Fig. 18) is shown in Fig. 19. A region of negative $\Delta T / T$ is present ahead of the crack tip which is introduced by bending of the upper face sheet.

$\Delta T / T$ results from the set-up with the G1 lens before and after motion compensation are shown in Fig. 20. The importance of compensating for specimen motion is highlighted even more clearly at these high spatial resolutions, as is evident by comparing the two images. In Fig. 20 (a) the position marks appear twice as does the crack path. In Fig 20 (b) the through-thickness $\Delta T / T$ gradient from compression to tension in the upper face sheet is clearly identifiable, as is the stress concentration at the crack tip, both in the face sheet and the foam, including a small area but clearly identifiable area of compression in the foam. The textured nature of the foam is clear in $\Delta T / T$.

The TSA results presented above have shown that effects of motion on the TSA can be effectively eliminated using the motion compensation technique. This enables the stress state to be assessed reliably, offering the opportunity to characterise interfacial cracks in sandwich structures. Furthermore the high spatial resolution TSA data obtained from the G1 lens allow small scale stresses to be investigated. This is important for studying the interfacial fracture behaviour when crack arresting devices [21] are introduced at the face sheet/core interface as the complex material combinations at the interface require the local stresses introduced at the crack arresting devices to be investigated experimentally. 


\section{Conclusions}

A motion compensation method based on DIC has been proposed for TSA. This method enables the use of a white light camera with high spatial resolution to capture the images for DIC thereby increasing the accuracy of the motion compensation, especially when the displacement field contains sharp gradients and discontinuities. The feasibility of the motion compensation method for TSA was investigated by studying the thermoelastic response around the edge of the hole using two lenses with different resolutions. It has been shown that the motion compensation method can significantly improve the accuracy of TSA measurements, especially when a magnifying lens is used. The motion compensation method has also been shown to make a significant improvement when obtaining TSA data from a DCB sandwich specimen. It is shown that the motion compensation method can be used to correct complex and non-uniform motion for TSA.

The requirement for image contrast to perform the DIC conflicts with the requirement for a uniform emissivity to perform the TSA. It has been demonstrated that by using a white light camera for DIC, a high contrast speckle can be used that has a uniform emissivity in the IR spectrum. This enables high spatial resolution and high fidelity displacement fields to be obtained without any need to reapply the surface preparation between DIC and TSA data capture or to interpolate across low emissivity speckles in the TSA data. Thereby this approach to motion compensation enables monitoring of fatigue crack growth, and other applications with complex and time varying motion fields.

\section{Acknowledgements}

The work presented was co-sponsored by the University of Southampton and the Danish Council for Independent Research | Technology and Production Sciences (FTP), under the research project 'Enhanced Performance of Sandwich Structures by Improved Damage Tolerance' ('SANTOL'). The financial support received is gratefully acknowledged. The foam material provided by DIAB AB Sweden is gratefully appreciated. 
Wang W., Fruehmann, R.K. and Dulieu-Barton, J.M., “Application of digital image correlation to address complex motions in thermoelastic stress analysis", Strain, 51, 2015, 405-418. doi: 10.1111/str.12151

\section{References}

[1] Dulieu-Barton, J M (2012). Thermoelastic stress analysis. In: Rastogi, P and Hack, E Optical Methods for Solid Mechanics. Weinheim: Wiley-VCH. p345-365.

[2] Stanley P, Chan W K 1985 Quantitative stress analysis by means of the thermoelastic effect J. Strain Anal. 20 129-137.

[3] Sutton, M A, Orteu, J and Schreier, HW (2009). Image correlation for shape, motion and deformation measurements. New York: Springer.

[4] Sakagami T, Yamaguchi N, Kubo S and Nishimura T 2008 A new full-field motion compensation technique for infrared stress measurement using digital image correlation J. Strain Anal. 43 539-549.

[5] Silva M L and Ravichandran G 2011 Combined thermoelastic stress analysis and digital image correlation with a single infrared camera J. Strain Anal. 46 783-793.

[6] Pottier T, Moutrille M -P, Le Cam J. -B, Balandraud X, Grediac M 2009 Study on the use of motion compensation techniques to determine heat sources. Application to large deformations on cracked rubber specimens Exp Mech. 49 561-574.

[7] Aviles F and Carlsson L A 2008 Analysis of the sandwich DCB specimen for debond characterization Eng. Fract. Mech. 75 153-168.

[8] Dulieu-Barton J M and Worden K 2003 Genetic identification of crack-tip parameters using thermoelastic isopachics Meas. Sci. Technol 14 176-183.

[9] Diaz F A, Patterson E A, Tomlinson R A and Yates J R 2004 Measuring stress intensity factors during fatigue crack growth using thermoelasticity Fatigue Fract. Eng. Mater. Struct. 27 571-583.

[10] Bodelot L, Charkaluk E, Sabatier L, Dufrenoy P 2011 Experimental study of heterogeneities in strain and temperature fields at the microstructural level of polycrystalline metals through fully-coupled full-field measurements by digital image correlation and infrared thermography Mech Mater. 43 654-670. 
Wang W., Fruehmann, R.K. and Dulieu-Barton, J.M., “Application of digital image correlation to address complex motions in thermoelastic stress analysis", Strain, 51, 2015, 405-418. doi: 10.1111/str.12151

[11] Seghi R, Bodelot L, Charkaluk E, Dufrenoy P 2012 Numerical and experimental estimation of thermomechanical fields heterogeneity at the grain scale of $316 \mathrm{~L}$ stainless steel Comput. Mater. Sci. 53 464-473.

[12] Seghi R, Witz J -F, Bodelot L, Charkaluk E, Dufrenoy P 2013 An improved Lagrangian thermography procedure for the quantification of the temperature fields within polycrystals $Q I R T 10$ 74-95.

[13] Samaca Martinez J R, Toussaint E, Balandraud X, Le Cam J -B, Berghezan D 2015 Heat and strain measurements at the crack tip of filled rubber under cyclic loadings using full-field techniques Mech Mater. 81 62-71.

[14] Toussaint E, Balandraud X, Le Cam J -B, Grediac M 2012 Combining displacement, strain, temperature and heat source fields to investigate the thermomechanical response of an elastomeric specimen subjected to large deformations Polym Test 31 916-925.

[15] Samaca Martinez J R, Balandraud X, Toussaint E, Le Cam J -B, Berghezan D 2014 Thermomechanical analysis of the crack tip zone in stretched and crystallisable natural rubber by using infrared thermography and digital image correlation Polymer $556345-6353$.

[16] Robinson A F, Dulieu-Barton J M, Quinn S and Burguete R L 2010 Paint coating characterization for thermoelastic stress analysis of metallic material Meas. Sci. Technol 21 (8).

[17] Yaofeng S and Pang J H L 2007 Study of optimal subset size in digital image correlation of speckle pattern images Opt Lasers Eng 45 967-974.

[18] ANSYS 12.1 manual, ANSYS Inc Canonsburg, 2009.

[19] Crammond G, Boyd S W, Dulieu-Barton J M 2013 Speckle pattern quality assessment for digital image correlation Opt Lasers Eng 51 (12) 1368-1378.

[20] Crammond G, Dulieu-Barton J M, Boyd S W 2015 A point-wise approach to the analysis of complex composite structures using digital image correlation and thermoelastic stress analysis, accepted for publication in Strain subject to minor correections. 
Wang W., Fruehmann, R.K. and Dulieu-Barton, J.M., "Application of digital image correlation to address complex motions in thermoelastic stress analysis", Strain, 51, 2015, 405-418. doi: 10.1111/str.12151

[21] Jakobsen J, Bozhevolnaya E and Thomsen O T 2007 New peel stopper concept for sandwich structures Compos. Sci. Technol. 67 3378-3385. 


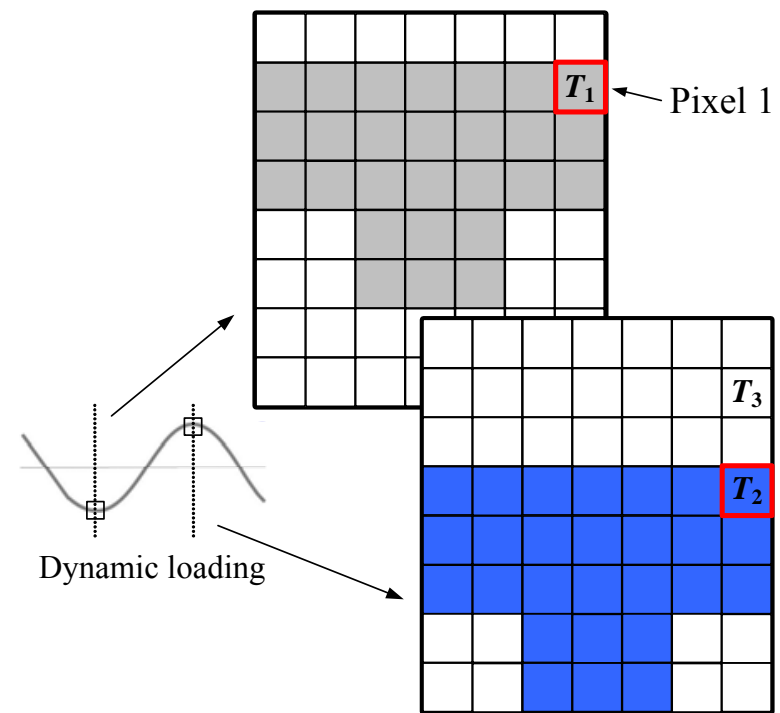

IR images
Background temperature

Specimen temperature at min load

Specimen temperature at max load

Fig. 1 Sample motion observed in IR images during cyclic loading

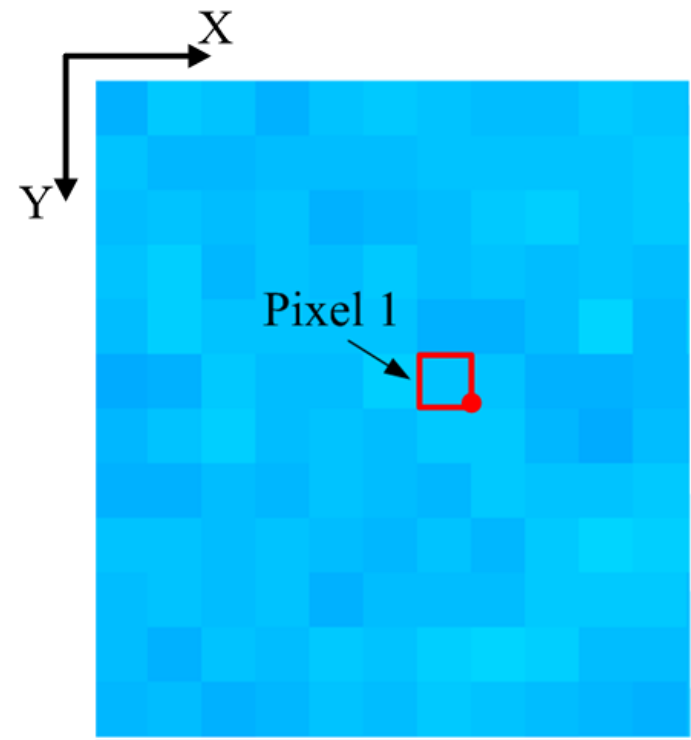

IR image

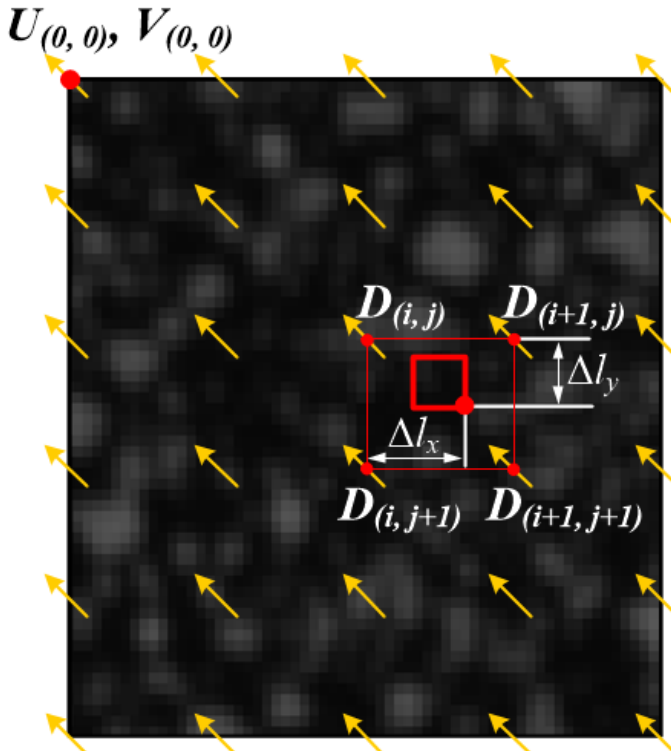

Displacement field

Fig. 2 A typical thermal image and its corresponding displacement 


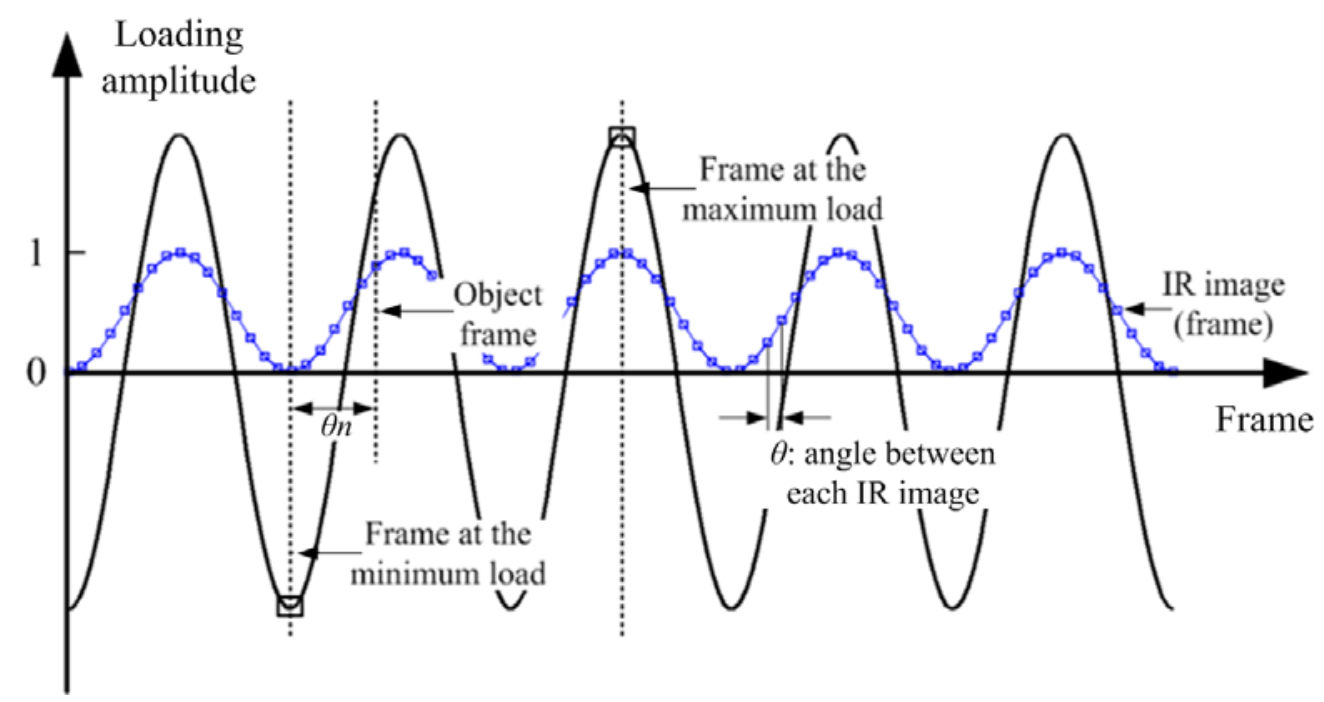

Fig. 3 IR images associated with the load signal and the periodic wave
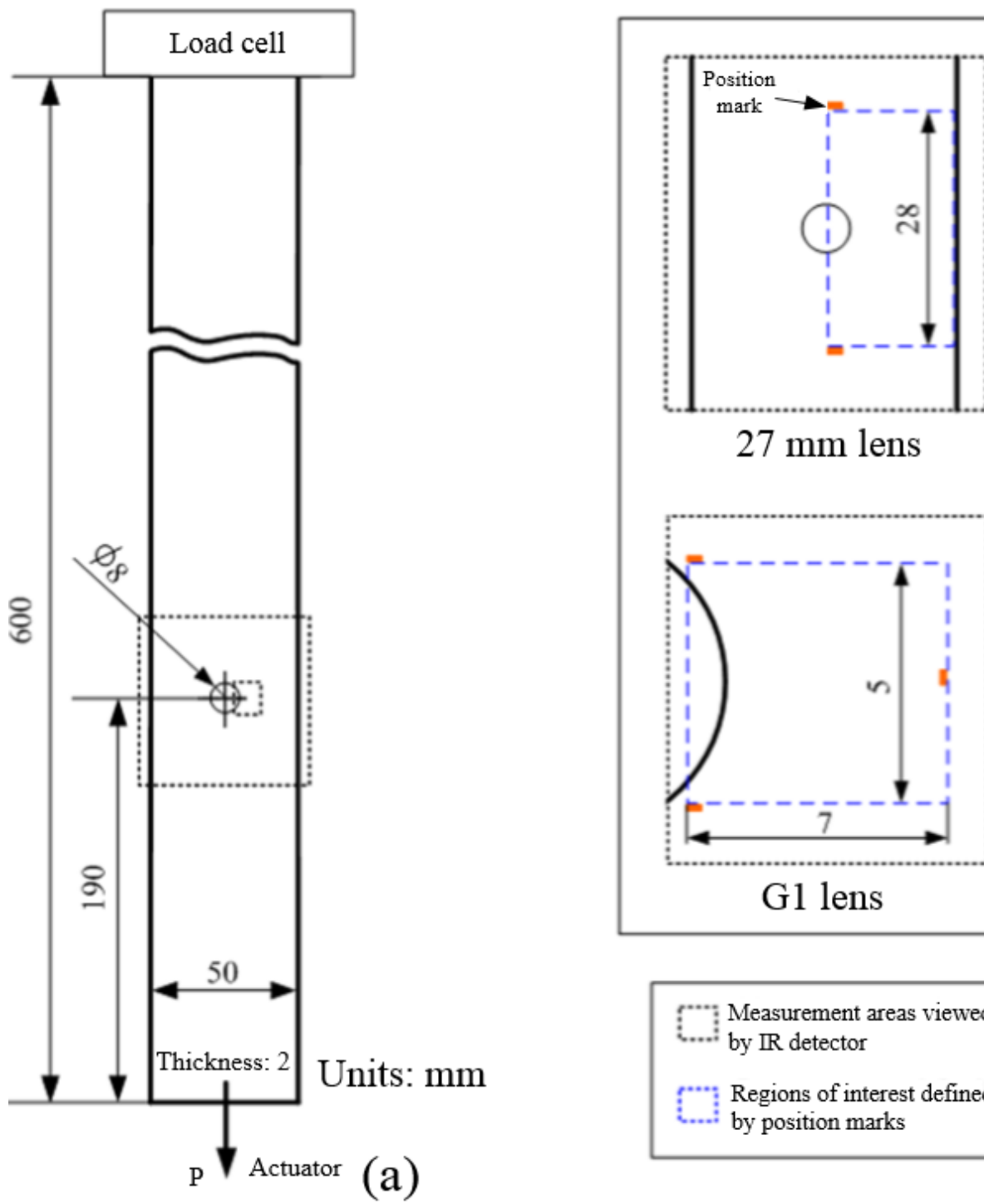

$27 \mathrm{~mm}$ lens
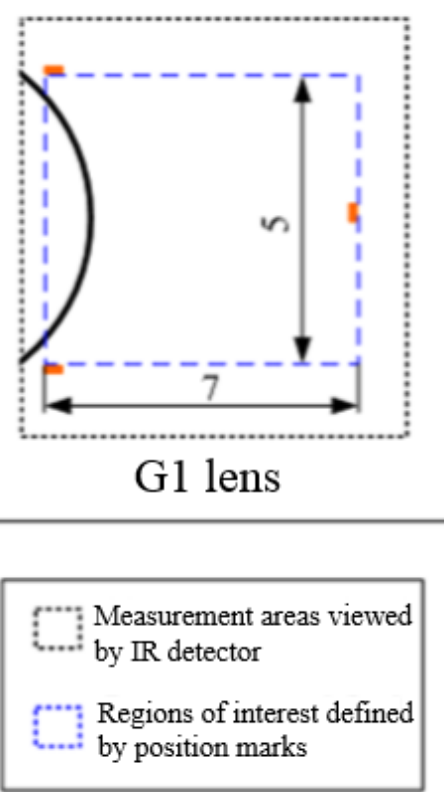

(b)

Fig. 4 Test specimen and setup: (a) dimensions of the test specimen (b) measurement areas viewed by the IR detector with different lenses and the corresponding regions of interest 

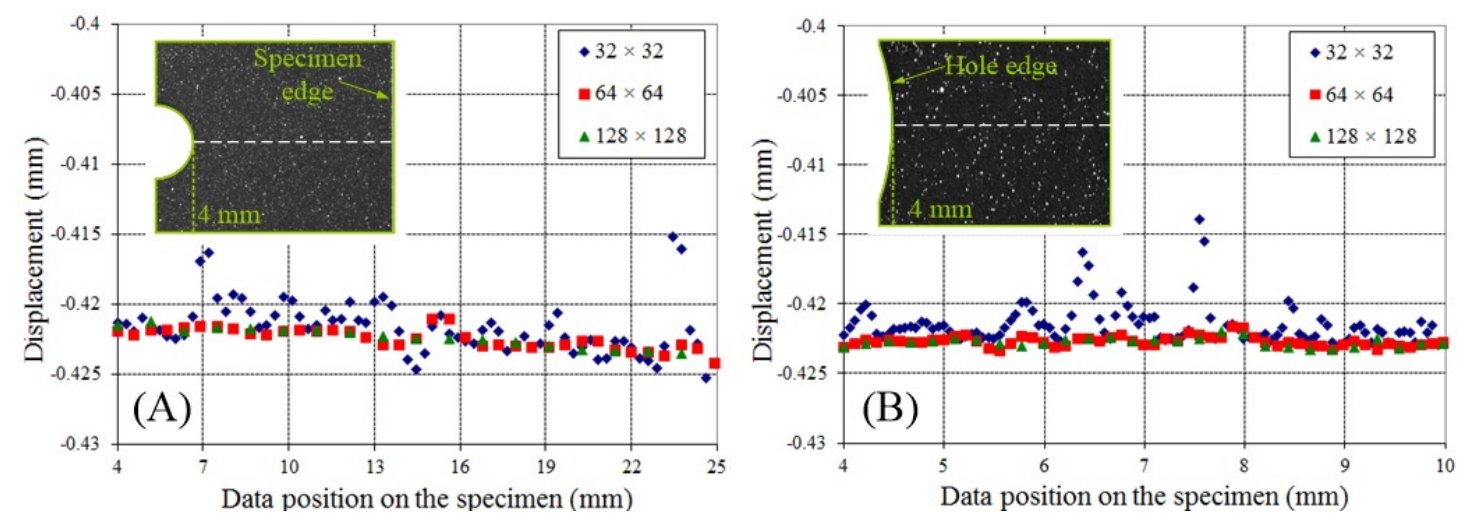

Fig. 5 Displacements derived with different subsets: (a) $105 \mathrm{~mm}$ lens (b) $65 \mathrm{~mm}$ lens

(a)

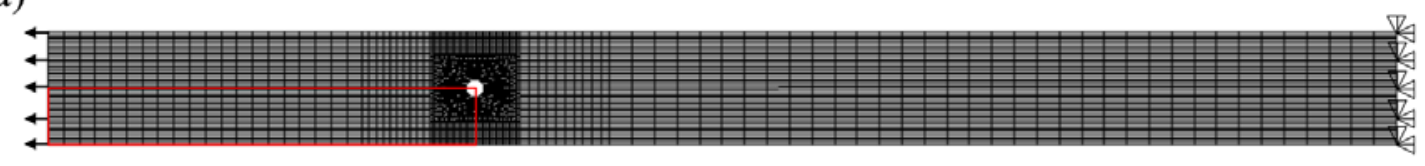

(b) Specimen edge loaded Local coordinates (the

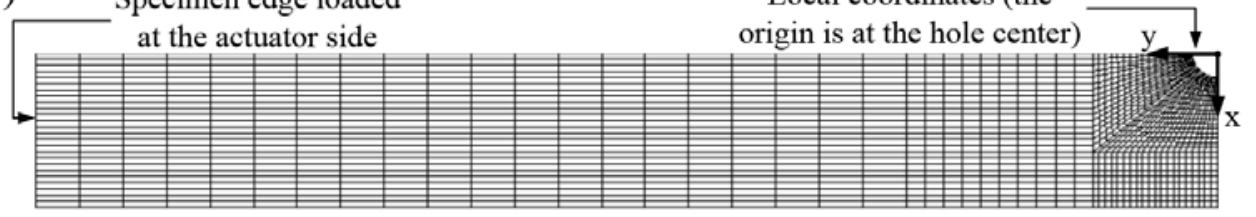

Fig. 6 (a) FE model of the aluminium plate specimen with the boundary conditions and (b) close-up details of the mesh around the hole 

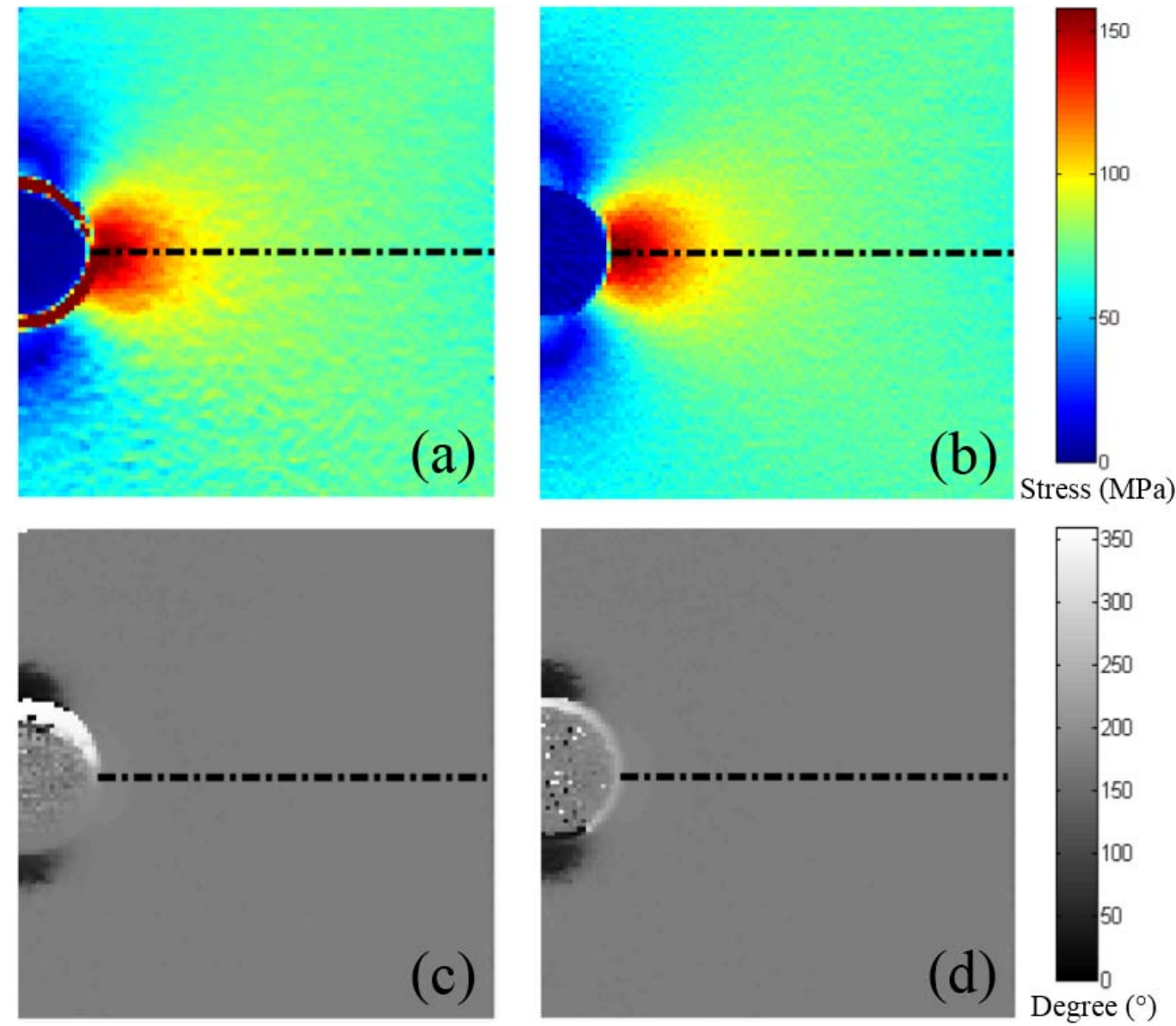

Fig. 7: The change in the in the sum of principal stresses and phase shift obtained by $27 \mathrm{~mm}$ lens: (a) stresses without MC, (b) stresses with MC, (c) phase shift without MC, (d) phase shift with MC
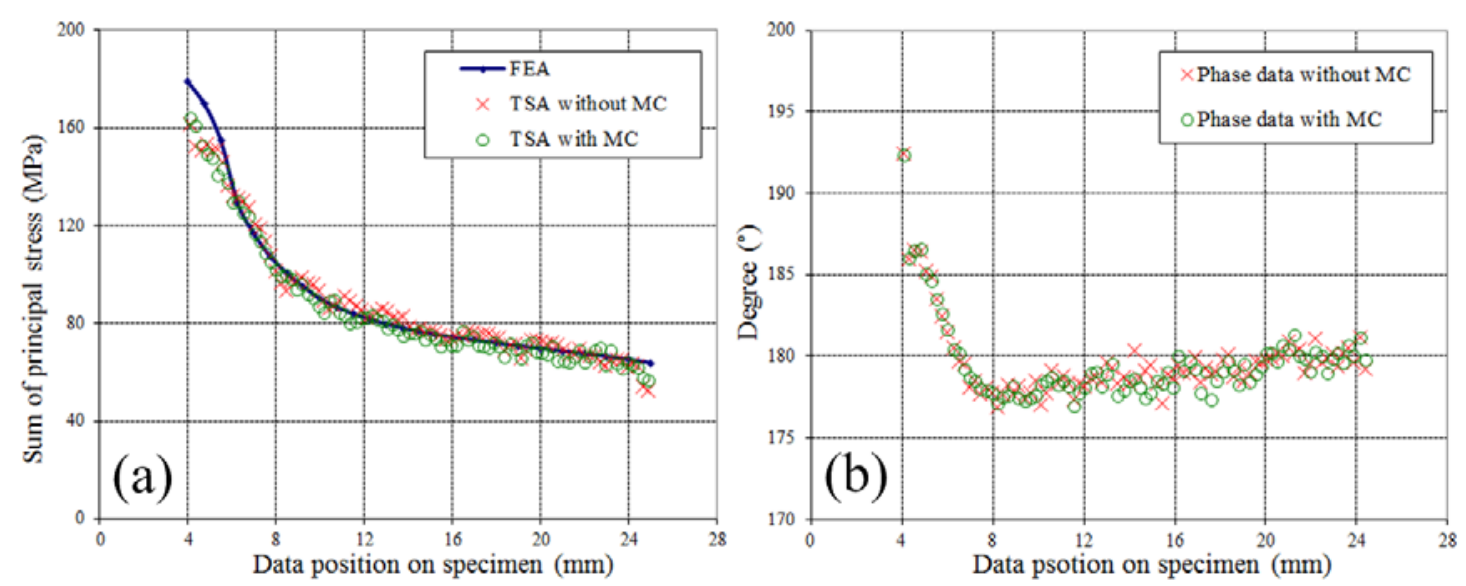

Fig. 8 Line plots of the TSA data and the FE results: (a) the comparison of the stresses from TSA with the FE analysis, (b) Phase data before and after MC 

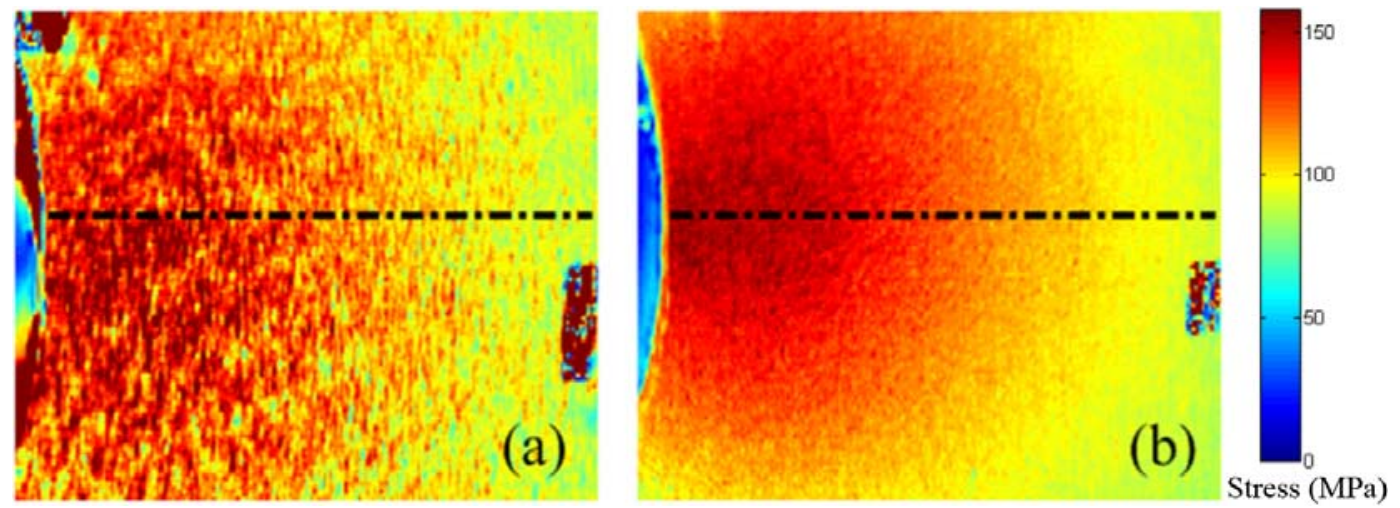

Fig. 9: The change in the sum of principal stresses obtained with the G1 lens: (a) without MC, (b) with MC
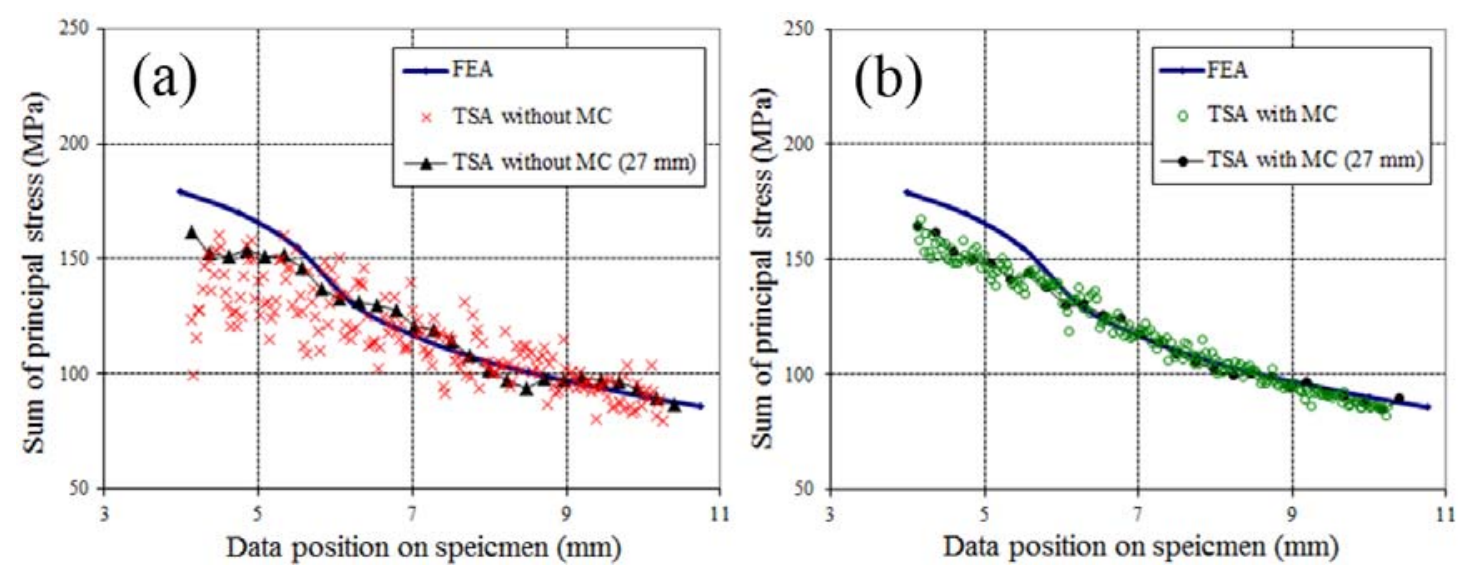

Fig. 10 Line plots of the FE results and TSA data obtained from both the G1 lens and the $27 \mathrm{~mm}$ lens

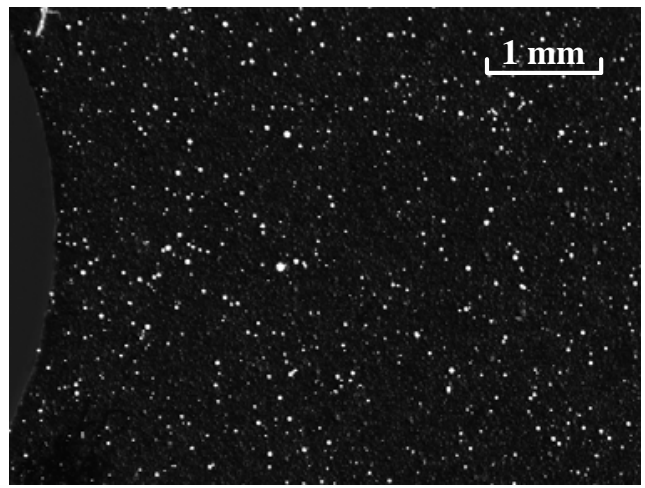

Fig. 11 Speckle pattern observed by the white light camera using $65 \mathrm{~mm}$ lens 


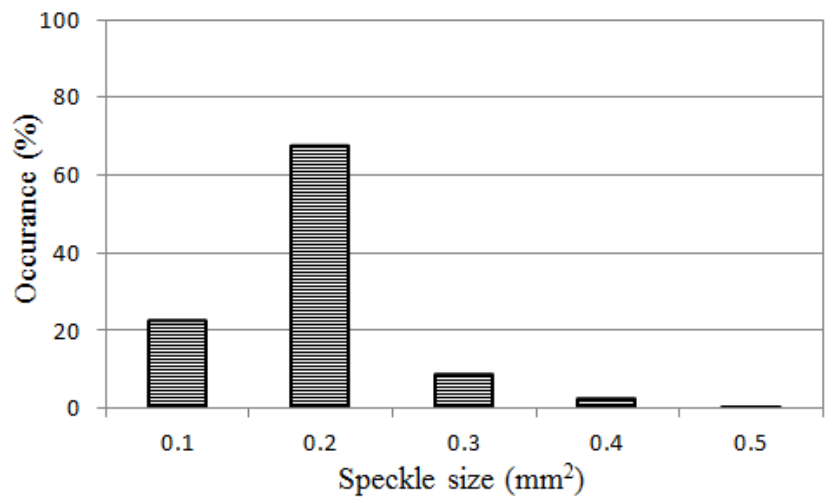

Fig. 12 The frequency distribution of the speckle size within the pattern
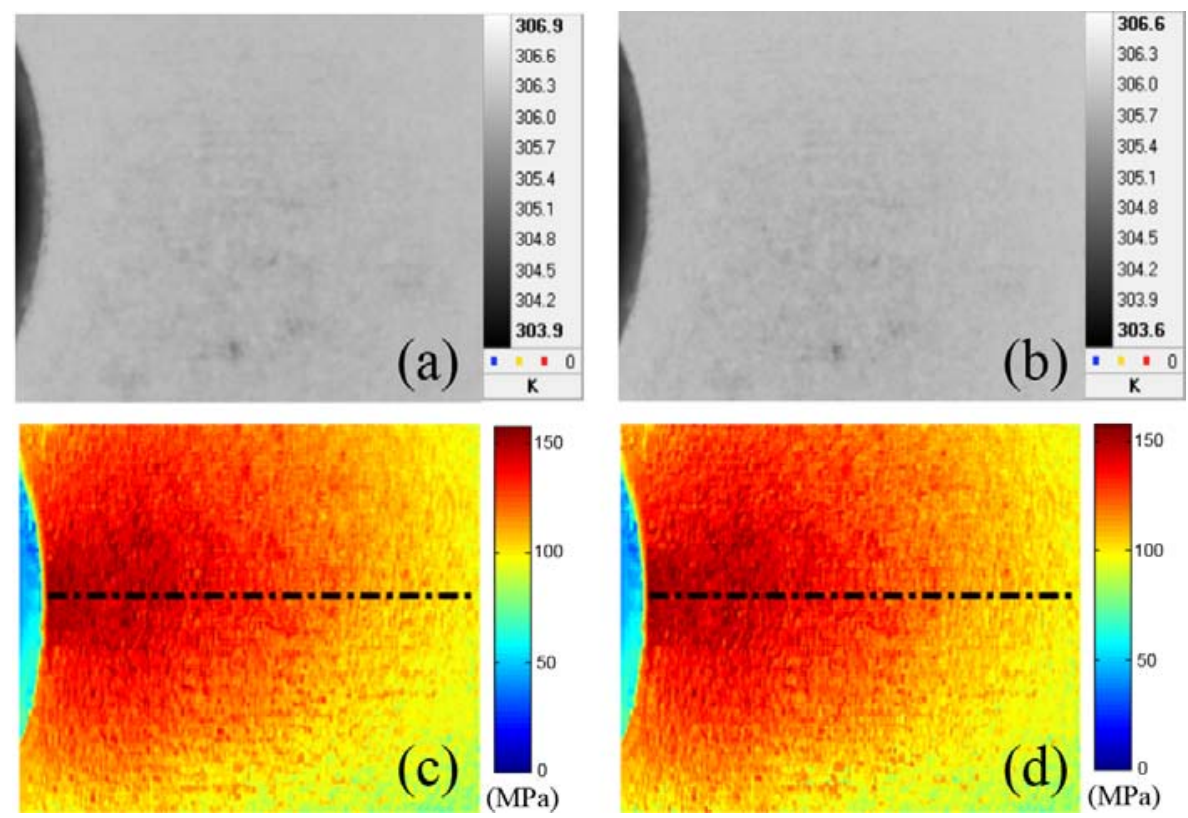

Fig. 13 Comparison of the IR images and TSA with different coatings: (a) IR image with black coating, (b) IR image with black-white speckle pattern, (c) TSA with black coating, (d) TSA with black-white speckle pattern 


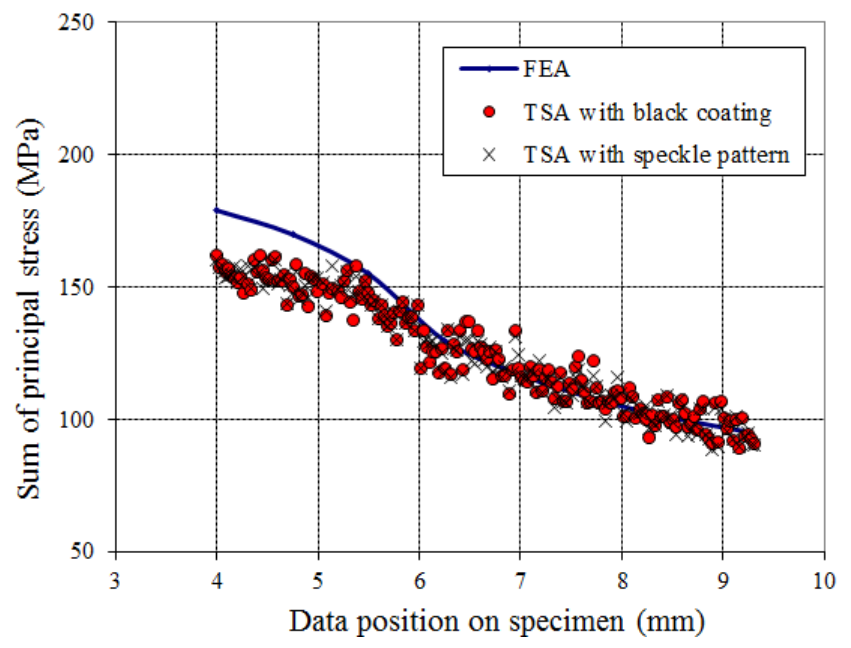

Fig. 14 Line plots of the TSA data from both black matt paint and speckle pattern coated specimen

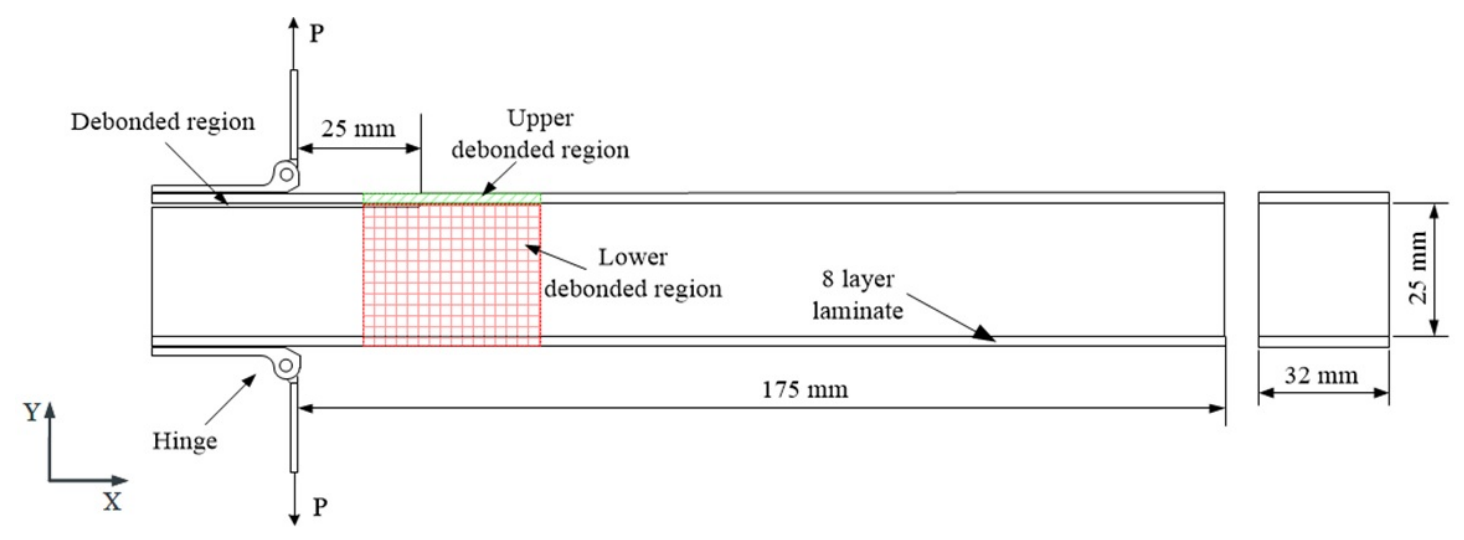

Fig. 15 DCB sandwich beam specimen 

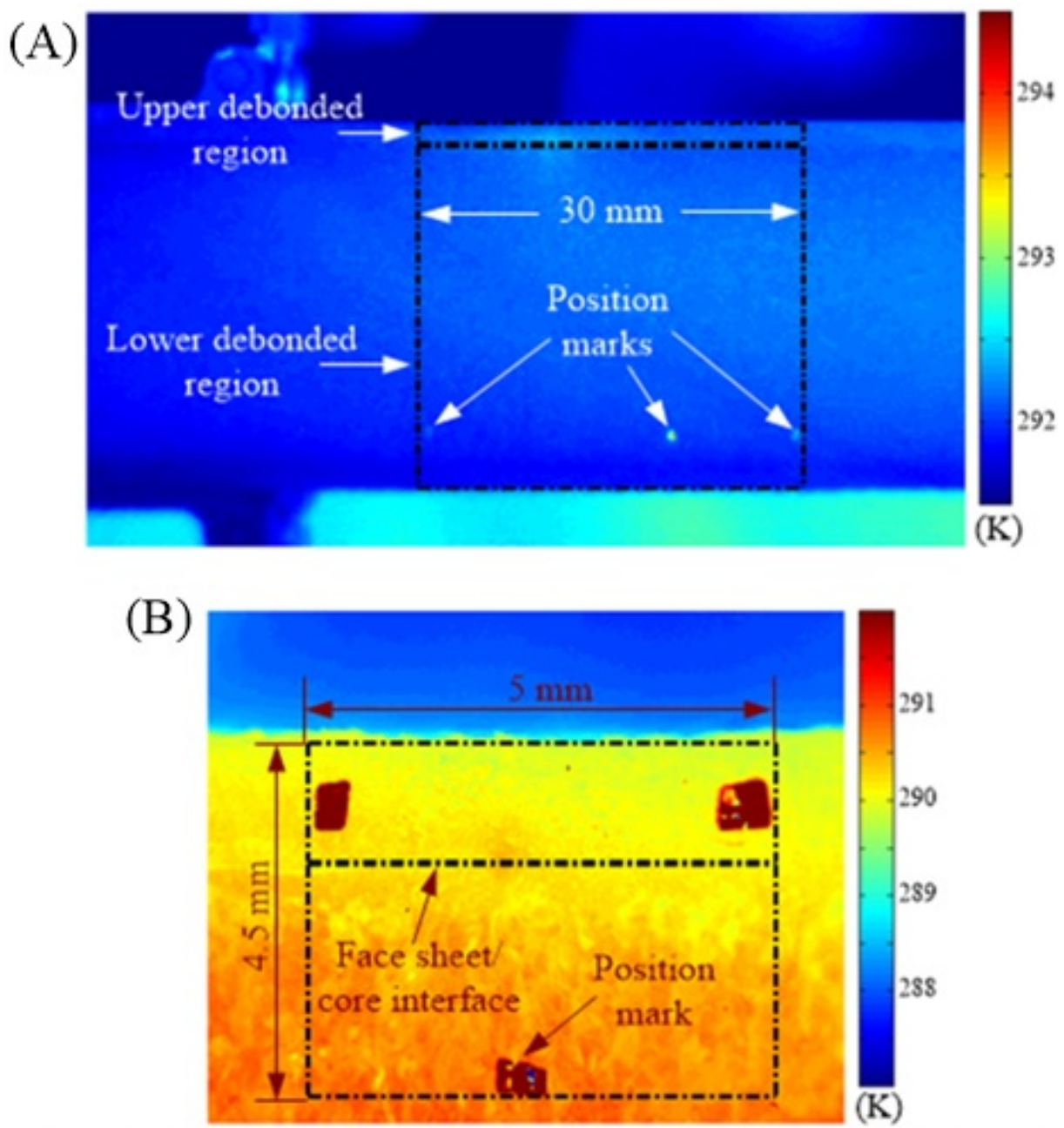

Fig. 16 IR images obtained using the: (a) $27 \mathrm{~mm}$ lens (b) G1 lens

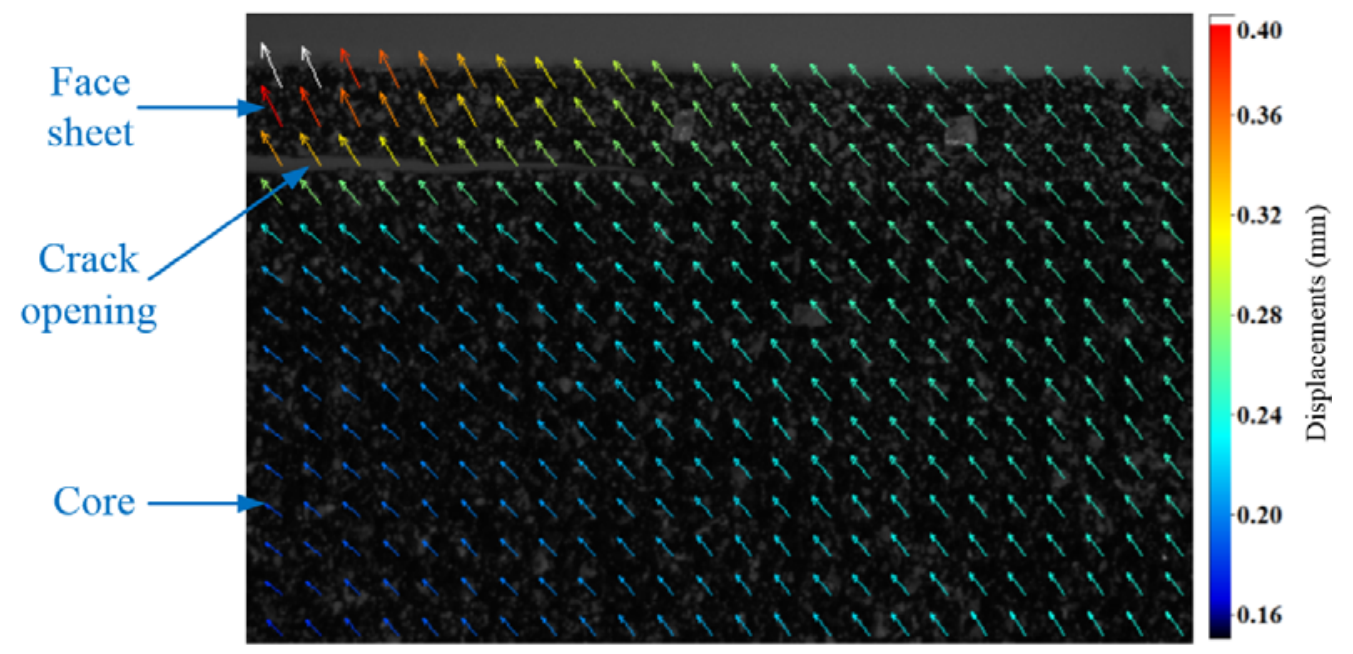

Fig.17 Displacement vectors around the crack tip in the sandwich structure specimen obtained using DIC 


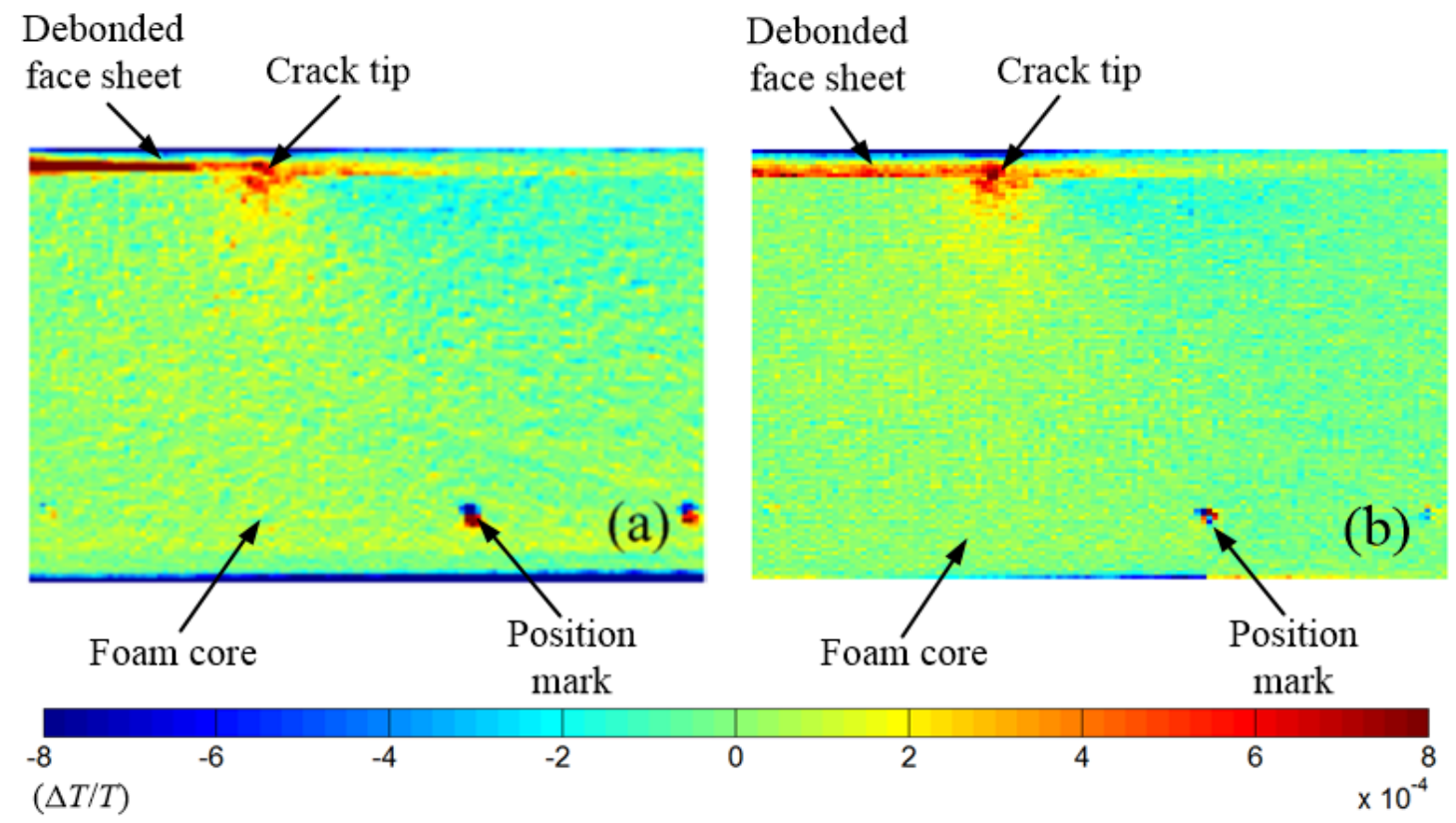

Fig.18 $\Delta T / T$ distribution in the sandwich structure specimen obtained with the $27 \mathrm{~mm}$ lens: (a) without MC (b) with MC

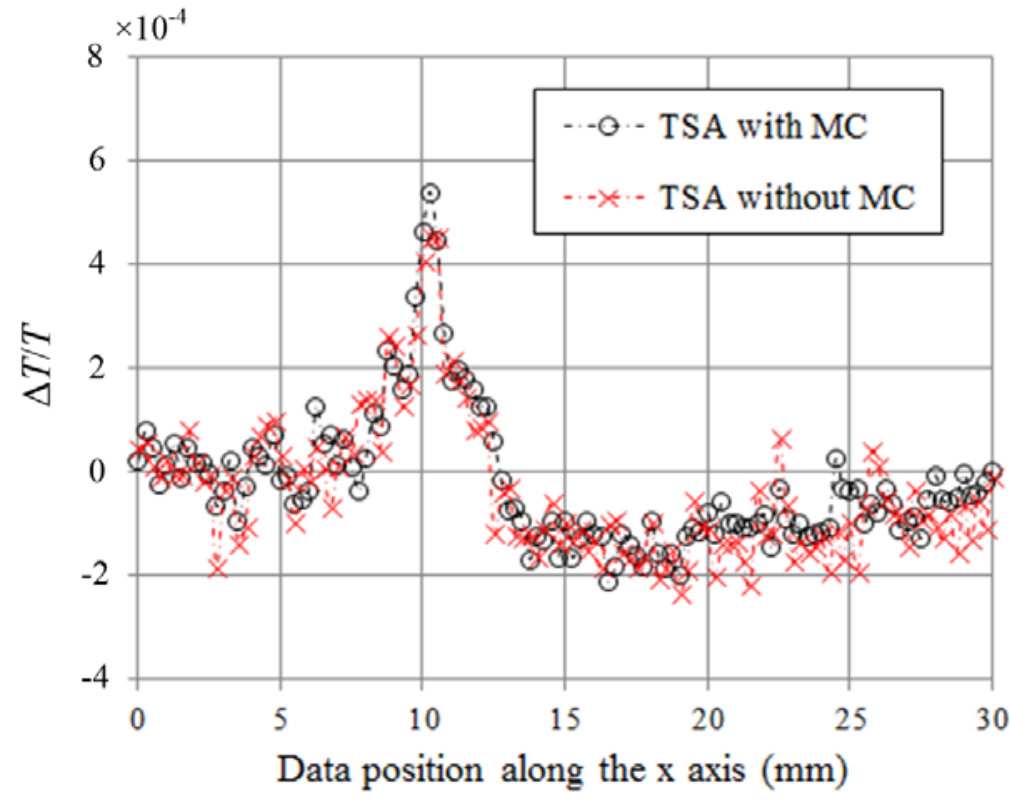

Fig.19 Line plot of $\Delta T / T$ along $\mathrm{x}$-direction taken $1 \mathrm{~mm}$ below the face sheet/core interface 


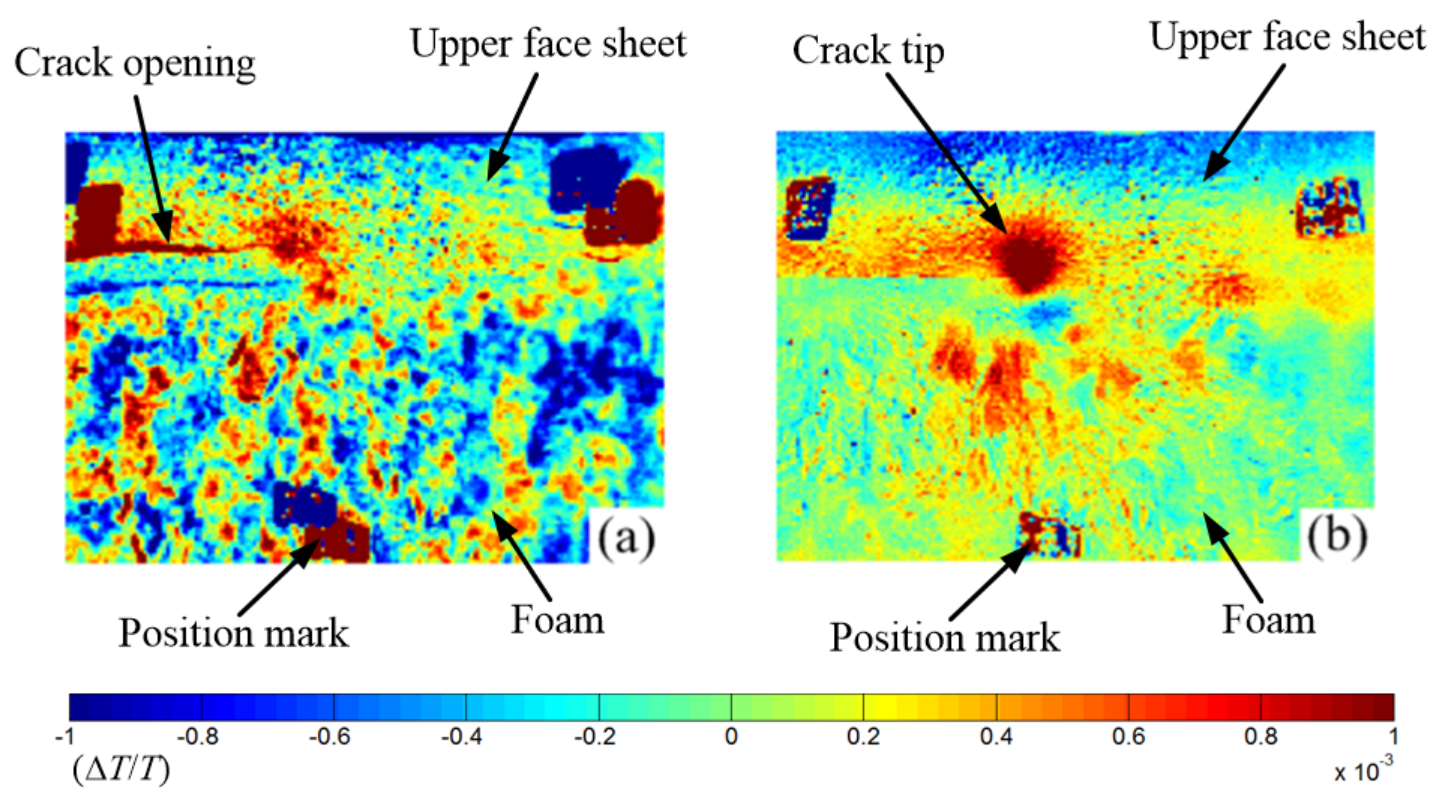

Fig.20 $\Delta T / T$ distribution on the DCB specimen obtained with the G1 lens: (a) without $\mathrm{MC}$ (b) with MC 